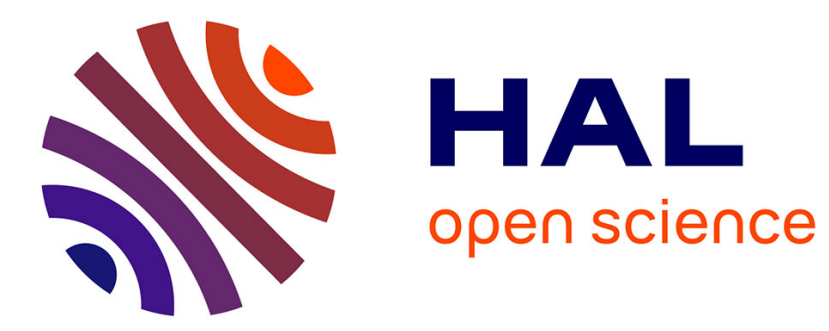

\title{
Structure sensitivity in third-harmonic generation microscopy
}

Delphine Débarre, Willy Supatto, Emmanuel Beaurepaire

\section{To cite this version:}

Delphine Débarre, Willy Supatto, Emmanuel Beaurepaire. Structure sensitivity in third-harmonic generation microscopy. Optics Letters, 2005, 30, pp.2134-2136. 10.1364/OL.30.002134 . hal-00681937

\section{HAL Id: hal-00681937 \\ https://hal.science/hal-00681937}

Submitted on 28 Oct 2013

HAL is a multi-disciplinary open access archive for the deposit and dissemination of scientific research documents, whether they are published or not. The documents may come from teaching and research institutions in France or abroad, or from public or private research centers.
L'archive ouverte pluridisciplinaire HAL, est destinée au dépôt et à la diffusion de documents scientifiques de niveau recherche, publiés ou non, émanant des établissements d'enseignement et de recherche français ou étrangers, des laboratoires publics ou privés. 


\title{
Structure sensitivity in third-harmonic generation microscopy
}

\author{
Delphine Débarre \\ Laboratory for Optics and Biosciences, Centre National de la Recherche Scientifique, Ecole Polytechnique, 91128 \\ Palaiseau, France \\ Willy Supatto \\ Laboratory of Physico-Chemistry, Centre National de la Recherche Scientifique, Institut Curie, 75005 Paris, France \\ Emmanuel Beaurepaire \\ Laboratory for Optics and Biosciences, Centre National de la Recherche Scientifique, Ecole Polytechnique, 91128 \\ Palaiseau, France \\ Received February 25, 2005

\begin{abstract}
We characterize experimentally the influence of sample structure and beam focusing on signal level in thirdharmonic generation (THG) microscopy. In the case of a homogeneous spherical sample, the dependence of the signal on the size of the sphere can be controlled by modifying the Rayleigh length of the excitation beam. More generally, the influence of excitation focusing on the signal depends on sample geometry, allowing one to highlight certain structures within a complex system. We illustrate this point by focusing-based contrast modulation in THG images of Drosophila embryos. () 2005 Optical Society of America

OCIS codes: $180.0180,190.4160,190.4180,180.6900,170.3880,180.5810$.
\end{abstract}

Owing to the success of multiphoton-excited fluorescence microscopy, ${ }^{1}$ biological imaging based on nonlinear coherent processes such as second-harmonic generation, ${ }^{2}$ third-harmonic generation (THG), ${ }^{3,4}$ or coherent anti-Stokes Raman scattering ${ }^{5}$ is receiving increasing interest. In these microscopies, the signal is a coherent superposition of the fields radiated by different objects within the excitation volume. Therefore the phase relation between the emitters is critical, and the signal depends on both the spatial organization of the scatterers and the structure of the driving field. ${ }^{2,6}$ A specificity of THG microscopy is that the signal generated by a beam focused inside a homogeneous, normally dispersive medium vanishes as a consequence of the Gouy phase shift experienced by the excitation beam near focus., 3,7 This effect makes THG imaging sensitive to inhomogeneities and has interesting consequences that need to be investigated to develop practical applications. In this Letter we look into THG as a function of beam focusing and sample geometry. First, we show how the signal obtained from a small object depends on both object size and beam focusing, in agreement with theoretical calculations. Then we demonstrate the influence of the focusing conditions on the signal generated from different structures. Finally, we apply these observations to structure-based contrast modulation in THG images.

THG imaging was performed with a custom-built scanning microscope incorporating photon-counting photomultipliers (Electron Tubes) and an optical parametric oscillator (APE) providing $200 \mathrm{fs}$ pulses with linear polarization at $1180 \mathrm{~nm}$. The THG signal was detected in the transmitted direction through a
0.9 numerical aperture (NA) condenser and a $390 \mathrm{~nm}$ interference filter.

We used a $20 \times 0.95$ NA water objective (Olympus) whose back aperture was underfilled to control the beam focusing. To this end, we introduced a pair of lenses forming a 2:1 beam expander before the microscope. By varying the distance $d$ between the lenses, we controlled the divergence of the beam and therefore its diameter at the back of the objective, resulting in variable focusing after the objective. We calibrated the Rayleigh length $z_{R}$ of the focused beam as a function of $d$ by measuring the full width at halfmaximum $\delta_{z}$ of the axial profile of the THG signal obtained when scanning through the upper face of a glass coverslip perpendicular to the beam. For an unclipped beam with $\mathrm{NA} \leqslant 0.9$, the shape of the profile is close to a squared Lorentzian with $\delta_{z} \approx 1.3 z_{R}$. Since $\delta_{z}$ is a relevant measure of the axial resolution for THG imaging, we directly use it as a calibration parameter in this Letter. In our experiments, we varied $\delta_{z}$ from 2 to $6 \mu \mathrm{m}$ corresponding to an effective numerical aperture in the range of $0.9-0.5$. We corrected the small focus shift resulting from changes in beam divergence by adjusting the objective position accordingly.

First, we compared the THG signal obtained from individual polystyrene beads of different sizes for a given value of $\delta_{z}$ [Figs. 1(d) and 1(e)]. We recorded images of monodisperse beads (Sigma) embedded in a $3 \%$ agarose gel. To reduce the imprecision due to excitation intensity fluctuations, we used samples containing two different bead sizes in the range of $0.1-3 \mu \mathrm{m}$ at a concentration of $\sim 10^{-3}$ beads $/ \mu \mathrm{m}^{3}$. We imaged small regions $(30 \mu \mathrm{m} \times 30 \mu \mathrm{m} \times 100 \mu \mathrm{m})$ in 

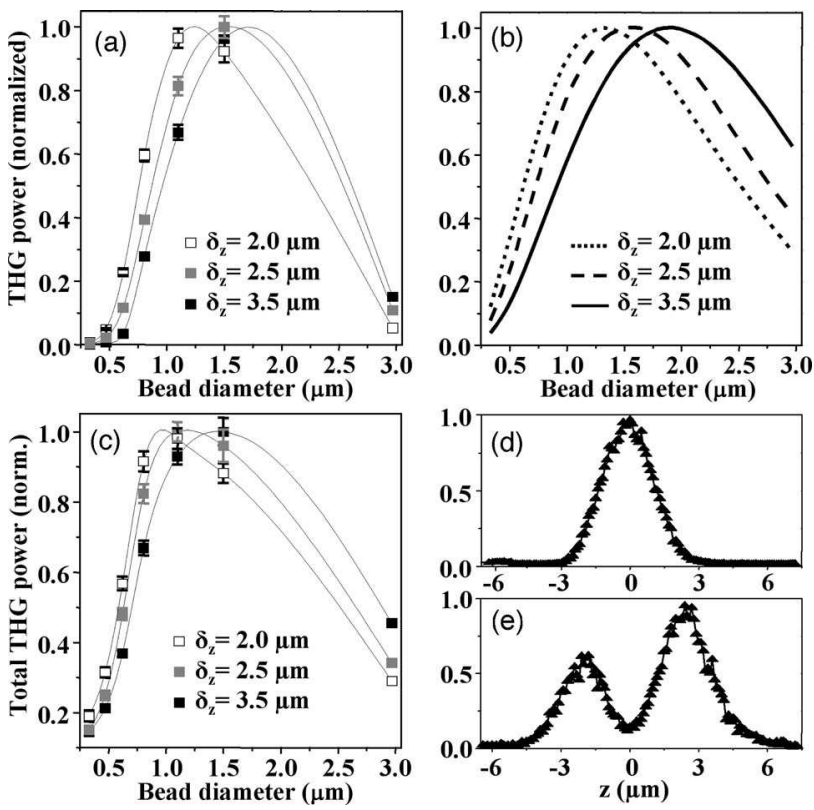

Fig. 1. (a) Measured THG power from a polystyrene bead centered at the beam focus as a function of bead diameter for three focusing conditions corresponding to $\delta_{z}=2,2.5$, and $3.5 \mu \mathrm{m}$ (respectively represented by white, gray, and black squares. Solid curves are a guide for the eye). (b) Theoretical THG power from a bead at the beam focus, assuming a refractive index of 1.57 at $1180 \mathrm{~nm}$ and 1.61 at 393 nm for polystyrene. (c) Total THG power from a bead during a volume scan, normalized to the bead volume. (d), (e) Axial THG profile through a (d) 0.6 and a (e) $3.0 \mu \mathrm{m}$ bead, with $\delta_{z}=2 \mu \mathrm{m}$.

each sample and measured the signal obtained from the center of each bead in this volume. We then calculated the ratio of intensities obtained for every pair of bead sizes. The uncertainty on the ratio was taken to be the standard error of the mean. Combining the ratios obtained for different bead sizes, we finally obtained the curves presented in Fig. 1(a).

Incorporating refractive-index dispersion into the formalism described by Cheng and Xie, ${ }^{6}$ we estimated the THG signal obtained from a bead centered at the beam focus. For simplicity, we assumed a focused Gaussian beam shape in our calculations. The signal field produced at a position $\mathbf{R}$ in the collection optics aperture can be expressed as ${ }^{6}$

$$
\begin{aligned}
\mathbf{E}^{\mathrm{THG}}(\mathbf{R}) \propto & \iiint_{V} \mathrm{~d} V\left(\mathbf{1}+\frac{\nabla \nabla}{k_{3}^{2}}\right) \frac{\exp \left(i k_{3}|\mathbf{R}-\mathbf{r}|\right)}{4 \pi|\mathbf{R}-\mathbf{r}|} \\
& \cdot \mathbf{P}^{\mathrm{THG}}(\mathbf{r}),
\end{aligned}
$$

where $V$ is the sample volume, $k_{3}$ is the thirdharmonic wave-vector amplitude, and $\mathbf{P}^{\mathrm{THG}}(\mathbf{r})$ is the induced nonlinear polarization at the third-harmonic frequency at position $\mathbf{r}$ within the sample. Integrating the squared field for $\mathbf{R}$ varying over the detection solid angle, we obtained theoretical curves [Fig. 1(b)] in qualitative agreement with the experiment. We attribute the difference between experiments and simulations principally to the fact that our calculations did not take into account the beam distortion caused by the refractive-index mismatch between the bead and the surrounding medium [see, e.g., the asymmetric profile in Fig. 1(e)].

We also measured the total THG signal generated during a volume scan of a bead, which is more relevant to imaging experiments [Fig. 1(c)]. Both data sets experimentally demonstrate that the signal reaches a maximum for a given bead diameter. Physically, the wave-vector mismatch introduced by the (tripled) Gouy shift and by index dispersion defines an axial interaction length over which constructive interference can occur. ${ }^{6}$ Interestingly, the size for which the maximum THG signal is obtained critically depends on $\delta_{z}$ because a reduction of the excitation NA (from 0.9 to 0.5 ) results in an axial spreading of the Gouy shift. As a consequence, the relative signal obtained from two objects of different sizes is a function of the focusing conditions. This remarkable property of THG microscopy could be exploited to realize a size spectroscopy of homogeneous spherical objects, e.g., by performing ratiometric measurements between different focusing conditions.

More generally, it is of practical interest to study the influence of focusing on the signal obtained from different types of structures within the sample. We first considered the case of a single interface perpendicular to the beam by imaging the upper side of a microscope coverslip [Fig. 2(a)]. As expected, the signal from the interface decreases with defocusing of the excitation beam [Fig. 2(c)]. We then imaged a fixed concentration $\left(5 \times 10^{-5}\right.$ volume fraction) of 0.6 or $3 \mu \mathrm{m}$ diameter beads [Fig. 2(b)], mimicking organelles in a biological system. In that case, the total signal increases with defocusing in the range explored here. Both effects are quantitatively accounted for by theory [Figs. 2(c) and 2(d)] and are due to the coherent summation of fields radiated from different points in the sample. When the beam is focused at the interface between two normally dispersive, semi-infinite media, the signal approximately scales as the third power of the intensity at the inter-
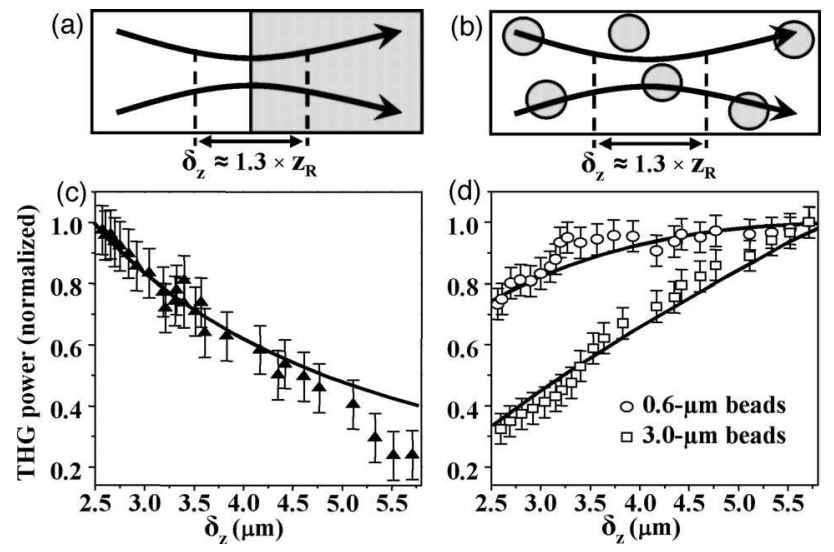

Fig. 2. THG power as a function of sample structure and focusing conditions. (a) Excitation beam scanned along an interface. (b) Excitation beam scanned in a gel of beads. (c) Total THG power obtained from a $30 \mu \mathrm{m} \times 30 \mu \mathrm{m}$ area in the geometry in (a). (d) Same measurement in the geometry in (b) for 0.6 (circles) and $3 \mu \mathrm{m}$ (squares) beads. Solid curves are theoretical calculations. 
face (i.e., as $\delta_{z}^{-3}$ ) times the squared illuminated area $\left(\sim \delta_{z}^{2}\right)$. Therefore THG scales as $\sim \delta_{z}^{-1}$. In contrast, two types of phenomena can cause an increase in total signal with defocusing when imaging a particlelike medium: (i) For beads smaller than $\delta_{z}[0.6 \mu \mathrm{m}$, Fig. $2(d)$, defocusing results both in an increase in the number of imaged objects and in individual bead oversampling, which counteracts the reduced excitation intensity. (ii) When imaging objects with size comparable to the excitation volume [ $3 \mu \mathrm{m}$, Fig. 2(d)], phase shift spreading caused by defocusing also extends the volume over which constructive interference occurs. In that case, the signal at the center of an individual bead increases with $\delta_{z}$ in the range explored here.

A practical consequence of these observations is that specific focusing conditions can be chosen to enhance certain structures in THG images. We illustrate this point by structure-selective imaging in early Drosophila embryos, depending on $\delta_{z}$. For a tightly focused beam, the main signal arises from the external membrane of the embryo [Fig. 3(c)], whereas for a more weakly focused beam the signal arises mostly from internal $\mu \mathrm{m}$-sized organelles ${ }^{8}$ [Fig. $\left.3(\mathrm{~d})\right]$. We measured the signal obtained from the center and from the outer membrane of the embryo as a function

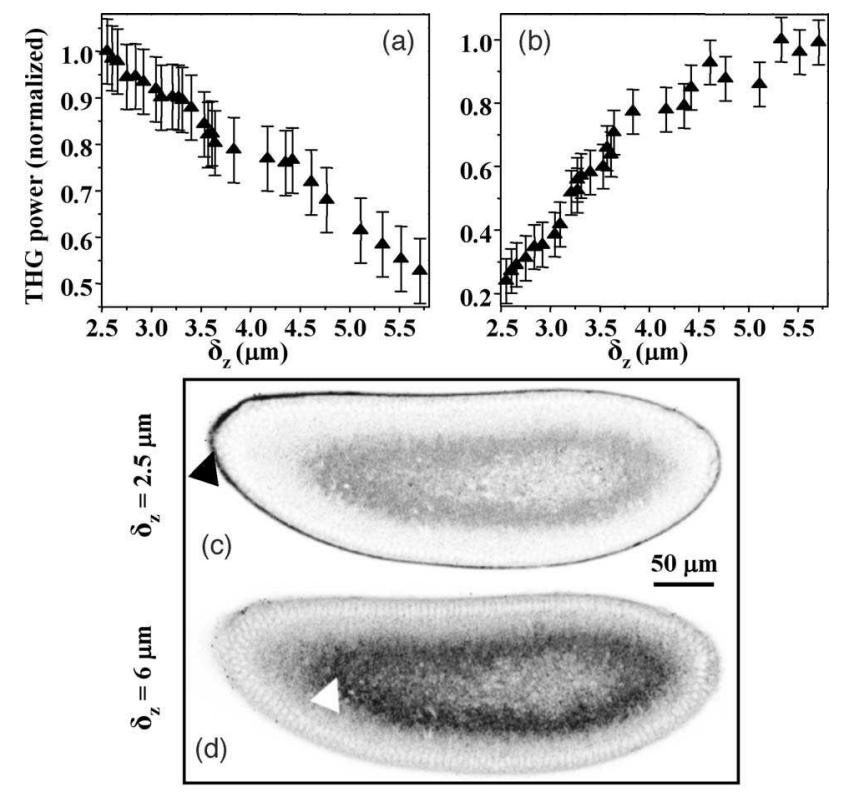

Fig. 3. Focusing-based contrast enhancement in THG images. (a) Total THG power obtained from the outer membrane of a Drosophila embryo [black arrowhead in (c)] as a function of $\delta_{z}$. (b) THG power obtained from internal organelles under the same conditions [white arrowhead in (d)]. (c), (d) Images recorded $50 \mu \mathrm{m}$ above the embryo equator for $\delta_{z}=2.5$ and $6 \mu \mathrm{m}$, normalized to the cubed excitation intensity and displayed with the same color code. Inverted contrast. Image acquisition time of $2.5 \mathrm{~s}$. of $\delta_{z}$ (Fig. 3). The membrane may be viewed as a tilted interface, whereas the center of the embryo can be compared with the images of polystyrene beads. Indeed, the dependence of the signal on $\delta_{z}$ is in both cases similar to that of Fig. 2, confirming the role of beam focusing on the images obtained.

One application of THG microscopy is the analysis of morphogenetic movements in developing embryos, ${ }^{8,9}$ and it relies on imaging of internal structures. For that purpose, tissue THG imaging is associated with correlation analysis that detects the motion of $\mu \mathrm{m}$-scale objects and therefore does not suffer from a loss of axial resolution in the $2-6 \mu \mathrm{m}$ range (corresponding to $\sim 0.6-1 \mu \mathrm{m}$ radial resolution). Comparing the two pictures in Fig. 3, it is clear that the image obtained with a weakly focused beam better reveals the internal structures of the embryo $(10 \times$ contrast improvement relative to the outer membrane), which makes it more suitable for correlation analysis. This counterintuitive result is strengthened by the fact that weak focusing results in an increased signal level inside the embryo, which can be exploited to reduce image acquisition time.

In conclusion, these experiments have demonstrated the influence of beam focusing on the relative signal obtained from different objects in THG microscopy. We have shown how this simple approach to phase-matching engineering can be exploited to enhance structures of interest in THG images in a practical case. Such effects should be of particular relevance when imaging tissues and complex systems.

We thank M. Joffre, M.-C. Schanne-Klein, and K. Kubarych for critical comments. This work was supported by the Délégation Générale pour l'Armement. E. Beaurepaire's e-mail address is emmanuel.beaurepaire@polytechnique.fr.

\section{References}

1. W. Denk, J. H. Strickler, and W. W. Webb, Science 248, 73 (1990).

2. L. Moreaux, O. Sandre, M. Blanchard-Desce, and J. Mertz, Opt. Lett. 25, 320 (2000).

3. Y. Barad, H. Eisenberg, M. Horowitz, and Y. Silberberg, Appl. Phys. Lett. 70, 922 (1997).

4. J. A. Squier, M. Müller, G. J. Brakenhoff, and K. R. Wilson, Opt. Express 3, 315 (1998).

5. A. Zumbusch, G. R. Holtom, and X. S. Xie, Phys. Rev. Lett. 82, 4142 (1999).

6. J.-X. Cheng and X. S. Xie, J. Opt. Soc. Am. B 19, 1604 (2002)

7. R. W. Boyd, Nonlinear Optics, 2nd ed. (Academic, 2003).

8. D. Débarre, W. Supatto, E. Farge, B. Moulia, M.-C. Schanne-Klein, and E. Beaurepaire, Opt. Lett. 29, 2881 (2004).

9. W. Supatto, D. Débarre, B. Moulia, E. Brouzés, J.-L. Martin, E. Farge, and E. Beaurepaire, Proc. Natl. Acad. Sci. U.S.A. 102, 1047 (2005). 